\title{
Adherence with Preventive Medication in Childhood Asthma
}

\author{
Scott Burgess, ${ }^{1}$ Peter Sly, ${ }^{2}$ and Sunalene Devadason ${ }^{3}$ \\ ${ }^{1}$ Department of Respiratory Medicine, Mater Children's Hospital, QLD 4101, Australia \\ ${ }^{2}$ Queensland Children's Medical Research Institute, QLD 4029, Australia \\ ${ }^{3}$ University of Western Australia, WA 6009, Australia
}

Correspondence should be addressed to Scott Burgess, scott.burgess2@mater.org.au

Received 29 November 2010; Accepted 12 February 2011

Academic Editor: Linjie Zhang

Copyright ( $\odot 2011$ Scott Burgess et al. This is an open access article distributed under the Creative Commons Attribution License, which permits unrestricted use, distribution, and reproduction in any medium, provided the original work is properly cited.

Suboptimal adherence with preventive medication is common and often unrecognised as a cause of poor asthma control. A number of risk factors for nonadherence have emerged from well-conducted studies. Unfortunately, patient report a physician's estimation of adherence and knowledge of these risk factors may not assist in determining whether non-adherence is a significant factor. Electronic monitoring devices are likely to be more frequently used to remind patients to take medication, as a strategy to motivate patients to maintain adherence, and a tool to evaluate adherence in subjects with poor disease control. The aim of this paper is to review non-adherence with preventive medication in childhood asthma, its impact on asthma control, methods of evaluating non-adherence, risk factors for suboptimal adherence, and strategies to enhance adherence.

\section{Introduction}

The aim of this paper is to review non-adherence with preventive medication in childhood asthma and its impact on asthma control. Methods of evaluating non-adherence and risk factors for sub-optimal adherence will be reviewed. Finally, the latest evidence for strategies to enhance adherence will be summarised.

\section{Compliance, Adherence, and Concordance}

"Compliance" describes the degree to which a patient takes a medication as it has been prescribed [1]. Compliance has largely been replaced by the term "adherence," which has fewer negative connotations [1]. It has been argued that both terms reflect a paternalistic model of care rather than a partnership. However, the alternative "concordance", which has been coined to reflect a therapeutic decision that incorporates the common goals of the physician and patient [1], has not been widely accepted.

\section{Management of Asthma and Preventive Medication}

Preventive medication is the corner stone of treatment for children with frequent intermittent or persistent asthma [2].
Preventive medication is taken on regular basis and has been shown to decrease inflammation within the lung and to improve disease outcomes [3].

\section{Importance of Adherence with Preventive Medication}

Non-adherence takes many forms, and the extent to which a patient is adherent with different asthma-related tasks may vary [4]. Patients may fail to attend appointments, fill prescriptions, miss doses of medication, or fail to use their inhalation device correctly. The incorrect use of an asthma device may be accidental (reflecting competence) or deliberate (contrivance) [5].

The impact of non-adherence depends upon the severity of the condition and the effectiveness of the treatment. Nonadherence is not necessarily inappropriate, particularly if the treatment is ineffective or harmful, so-called "intelligent non-adherence" [6]. The consequence of non-adherence with medication may also be dependent upon the pattern of non-adherence and pharmacological characteristics of the medication [7]. Sub-optimal adherence has been shown to result in poor disease control [8-10], an increased risk of hospital admission [11, 12], and asthma-related mortality [13-15]. If non-adherence is undetected, the physician may 
unnecessarily increase the dose of medication or add an additional treatment, increasing the cost and complexity of the regimen [16]. It has been estimated that nonadherence costs the US healthcare system $\$ 300$ billion each year [17]. Undetected non-adherence is also important in research trials, skewing results towards the null hypothesis [16].

\section{Measuring and Estimating Adherence with Preventive Medication}

Adherence may be measured by collecting data from the child or parent (direct questioning, questionnaires, and diaries), evaluating the amount of medication that has been used (counting returned pills, weighing canisters), reviewing pharmacy records, electronic monitoring devices (incorporated into the pill bottle or pressurised metered dose inhaler), or drug assays (serum or urine drugs levels) [18].

Electronic monitoring devices (EMDs) have been proposed as the Gold standard for measuring adherence [19]. EMDs record a time stamp which can be downloaded and compared with the prescribed regimen. Using these devices, it was discovered that some participants in a clinical trial were discharging large amounts of medication just prior to reviews, a process referred to as "dumping" [20]. EMDs do have limitations, most importantly they are expensive, some devices have been prone to electrical malfunction [18], and monitoring may itself alter the patients' behavior [18].

Compared with EMDs, parental report [21], questionnaires [22], diaries $[8,12,23,24]$, canister weights [21, $22,25-27$ ], and pill counts [28] have all been found to overestimate adherence. Even when parents were asked about their child's adherence using a nonjudgemental manner, prefaced by a normalising statement, the level of reported adherence did not accurately reflect data from an EMD [29]. Patients typically want their actions to be seen to be appropriate and responsible, a behaviour referred to as "social desirability" [30]. This strong social convention may result in seemingly honest people providing exaggerated reports of medication usage.

Healthcare professionals have been shown to be poor judges of adherence, identifying non-adherence no better than would be predicted by chance alone [29, 31-33]. The inability to predict adherence has been shown to be independent of seniority [34], professional stream (doctors versus nurses) [31], or how long the physician has known the patient [33].

\section{Adherence Rates in Clinical Studies}

Studies that have used EMDs to evaluate adherence with preventive asthma medication have reported average adherence rates of $50 \%$ to $77 \%[8,12,21,24,29,35]$. Even though these figures are low, they are likely to be higher than adherence within the general population as the most nonadherent patients do not normally enroll in trials [36] and simply being in a trial has been associated with increased adherence [37].

\section{Models of Adherence and Behaviour}

Models of adherence provide a framework for conceptualising adherence and have been the basis for recommended strategies to manage non-adherence in many review articles. The most commonly cited is the Health Beliefs Model.

According to the Health Beliefs Model, adherence is determined after a patient balances the costs of treatment (e.g., financial costs, and real and feared side effects) and threats posed by the health condition against the perceived benefits of treatment [4].

The Ecological Model takes into account contextual factors relating to the family (conflict, poverty, and education), social supports, stressors, and cultural factors. It reminds us that, for some families health may not be the first priority [4]. Other factors may be more pressing, including homelessness, substance abuse, and emotional disturbance [38]. The Ecological Model also takes into account individual factors, such as their beliefs, skills, motivation for change, and selfefficacy [4]. Individual factors also include developmental factors, for example a child's understanding of causality [39].

Evidence suggests that people do not necessarily mentally balance the pros and cons of a given decision. Rather, people typically make decisions instinctively, unconsciously, and are strongly influenced by their emotions and prejudices [40].

\section{Risk Factors and Improving Adherence}

8.1. Sex and Age. Studies examining adherence in the paediatric population [41], including studies using EMDs, $[21,42]$ have found no relationship between adherence and gender. The available data indicates that adherence generally falls with increasing age [21, 42-44]. The age at which parents allow children to assume responsibility for taking their own medication may reflect the parents' ability to supervise the child, not only the child's maturity, and this may result in unfounded confidence in their ability [45]. Parents and children may not be aware of the limits of their responsibilities. When the parent and their child believe the other is primarily responsible for remembering to take medication adherence is lower [46].

8.2. Disease Severity. Patients with frequent symptoms are not necessarily prompted to take their preventive medication. Observational studies have revealed that adherence is lower in those subjects with increased symptoms [35, 42, 44, 47]. Non-adherence should always be suspected in those with poorly controlled asthma.

8.3. Parent-Child Relationship, Parental Psychopathology, and Children's Behaviour. Young children are dependent upon their parents, and, thus, their domestic environment is critical in their disease management [48]. Adolescents report that reminders from parents to take medication are helpful $[49,50]$, even if they find them annoying [50]. Higher levels of adherence are reported by adolescents who described their parents as supportive and lower when their parents are described as overly anxious or controlling [51]. 
It is likely that a bidirectional relationship exists between parents' psychological function and their child's asthma control [52]. Children whose parents had poorer mental health scores were reported to experience more asthma symptoms, [53] exacerbations [53], higher rates of health care use [53], and reduced adherence [54]. A meta-analysis of 26 studies found that children with asthma, when compared with controls, have more behavioural difficulties, particularly in terms of internalising behaviours (e.g., anxiety, low mood, withdrawal, and somatic complaints) and to a lesser extent, externalising behaviours (aggressive behaviour or defiance) [55].

Asthmatic children with behavioural difficulties have been found to be less adherent with asthma medication [56].

8.4. Social Adversity, Ethnicity, and Family Function. Three relatively small, well-designed studies $[21,42,56]$ found no relationship between adherence and socioeconomic status. Adherence in these studies was based on either serum drug levels or EMDs. A number of studies in Western countries have found adherence to be lower in non-Caucasian participants $[21,42,43,57,58]$. However, minority status is a nonspecific risk factor and could reflect a number of potential barriers to adherence. A study involving African American parents from a low-income urban area reported that the greatest barrier to optimal treatment was parental health beliefs rather than access to healthcare or financial constraints [59]. Family dysfunction has been associated with reduced adherence $[12,21,56]$. Families without strong routines are less likely to fill prescriptions for asthma medication [60]. Non-adherence is greater in families with high levels of conflict and behavioural problems in their children [56].

8.5. Education, Knowledge and Communication. In order to be adherent patients require basic information about their medication, including how and when they are to be taken. However, numerous studies have failed to correlate asthma knowledge with the level of adherence [21, 42, 60-63], and simply providing information does not necessarily improve adherence [36]. Patients report higher levels of adherence with medication prescribed by physicians who communicate well $[57,58]$ and whose interactions were described as collaborative rather than authoritarian or generic [51]. In one study, there was $38 \%$ disagreement between parents of asthmatic children and their doctor about whether preventive medication had been prescribed during a consultation. Interestingly, discordance was greatest for those parents who had concerns about or negative perceptions of steroid medication [64]. In a landmark study, asthma outcomes were improved when paediatricians were taught to provide simple messages combined with basic communication and counseling strategies [65]. Thus, it is important not merely to educate parents, but to discover and address their specific concerns and confirm that you share common goals and tailor the management plan to the client.

8.6. The Delivery Device. There are limited studies examining the influence of the delivery device on adherence. A breath-activated nebulizer (Halolite) was not found to improve adherence compared with a standard metered dose inhaler and spacer [66]. Adherence was not different when two different dry powder devices were compared [67]. A randomised controlled trial which used an EMD to monitor adherence failed to demonstrate improved adherence by young children using a novel spacer (Funhaler) that included an incentive toy (whistle and spinning disk) [68], contrary to a pilot study that measured adherence using parental report [69]. It is intuitive to offer patients the opportunity to use the device they would prefer, but this does not guarantee increased adherence.

8.7. Frequency of Dosing. There is low-level evidence to suggest that adherence decreases as the dosing frequency of prescribed medication increases [24, 70, 71]. Thus, where possible, the regime should include as few doses as possible.

8.8. Type of Medication. Adherence appears to be higher with a once daily oral nonsteroidal asthma medication compared with an inhaled corticosteroid taken twice daily $[72,73]$. The combination of an ICS and long-acting beta-agonist also appears to be associated with superior adherence compared with ICS alone or both medications in separate inhalers [7476]. However, data on combined medications are not based on studies using EMDs.

8.9. Reminders. The most commonly reported reason patients give for failing to take medication is simply "forgetting" [49, 50, 77]. A randomised controlled study involving adult asthmatics found a significant improvement in adherence in those subjects whose medication was delivered via an EMD which flashed and beeped to remind them of the need to take their medication [78].

8.10. Measuring Adherence and Providing Feedback. Several studies have found increased adherence when data collected by an EMD were made available to study participants [27, 79-81]. Our group has recently completed a randomised controlled trial involving 26 children with poorly controlled asthma over a 4 month period. Average adherence was $21 \%$ higher in those who received feedback on their adherence at each study visit [82]. Larger studies are required to demonstrate the clinical efficacy of this strategy.

\section{Conclusion}

Suboptimal adherence with preventive medication is common and is associated with significant morbidity and healthcare costs. Non-adherence should be considered in all children with poorly controlled asthma. Adolescents, children from chaotic families, and children from socially disadvantaged groups are at increased risk of non-adherence. Unfortunately, patient report and your own impressions and knowledge of these risk factors may not help you determine whether non-adherence is the reason for a child's poor asthma control. When prescribing medication, it may be important to consider the complexity of the regimen in addition to the efficacy of the intervention. Treatment plans should be developed collaboratively, and it is important to explore the patient's concerns and prejudices. In the future, 
EMDs are likely to play a greater role as an aide to remind patients to take medication, a strategy to motivate patients to maintain adherence, and a tool to evaluate adherence in subjects with poor disease control.

\section{References}

[1] R. Horne, "Compliance, adherence, and concordance: implications for asthma treatment," Chest, vol. 130, no. 1, 2006.

[2] National Asthma Council Australia, Asthma Management Handbook 2006, Melbourne, Australia, 2006.

[3] E. W. Fong and R. H. Levin, "Inhaled corticosteroids for asthma," Pediatrics in Review, vol. 28, no. 6, pp. e30-e35, 2007.

[4] G. S. Liptak, "Enhancing patient compliance in pediatrics," Pediatrics in Review, vol. 17, no. 4, pp. 128-134, 1996.

[5] M. L. Everard, "Role of inhaler competence and contrivance in "difficult asthma"'” Paediatric Respiratory Reviews, vol. 4, no. 2, pp. 135-142, 2003.

[6] A. Dugdale, "Non-compliance or rational decision," The Lancet, vol. 342, no. 8884, pp. 1426-1427, 1993.

[7] J. Urquhart, "Defining the margins for errors in patient compliance with prescribed drug regimens," Pharmacoepidemiology and Drug Safety, vol. 9, no. 7, pp. 565-568, 2000.

[8] H. Milgrom, B. Bender, L. Ackerson, P. Bowry, B. Smith, and C. Rand, "Noncompliance and treatment failure in children with asthma," Journal of Allergy and Clinical Immunology, vol. 98, no. 6, pp. 1051-1057, 1996.

[9] B. Bender and L. Zhang, "Negative affect, medication adherence, and asthma control in children," Journal of Allergy and Clinical Immunology, vol. 122, no. 3, pp. 490-495, 2008.

[10] L. K. Williams, M. Pladevall, H. Xi et al., "Relationship between adherence to inhaled corticosteroids and poor outcomes among adults with asthma," Journal of Allergy and Clinical Immunology, vol. 114, no. 6, pp. 1288-1293, 2004.

[11] G. A. Ordoñez, P. D. Phelan, A. Olinsky, and C. F. Robertson, "Preventable factors in hospital admissions for asthma," Archives of Disease in Childhood, vol. 78, no. 2, pp. 143-147, 1998.

[12] B. Bender, H. Milgrom, C. Rand, and L. Ackerson, "Psychological factors associated with medication nonadherence in asthmatic children," Journal of Asthma, vol. 35, no. 4, pp. 347353, 1998.

[13] C. F. Robertson, A. R. Rubinfeld, and G. Bowes, "Pediatric asthma deaths in Victoria: the mild are at risk," Pediatric pulmonology, vol. 13, no. 2, pp. 95-100, 1992.

[14] G. Birkhead, N. J. Attaway, R. C. Strunk, M. C. Townsend, and S. Teutsch, "Investigation of a cluster of deaths of adolescents from asthma: evidence implicating inadequate treatment and poor patient adherence with medications," Journal of Allergy and Clinical Immunology, vol. 84, no. 4, pp. 484-491, 1989.

[15] R. C. Strunk, "Asthma deaths in childhood: identification of patients at risk and intervention," Journal of Allergy and Clinical Immunology, vol. 80, no. 3, pp. 472-477, 1987.

[16] J. A. Cramer, "Feedback on medication dosing enhances patient compliance," Chest, vol. 104, no. 2, pp. 333-334, 1993.

[17] B. G. Bender and C. Rand, "Medication non-adherence and asthma treatment cost," Current Opinion in Allergy and Clinical Immunology, vol. 4, no. 3, pp. 191-195, 2004.

[18] K. A. Riekert and C. S. Rand, "Electronic monitoring of medication adherence: when is high-tech best?" Journal of Clinical Psychology in Medical Settings, vol. 9, no. 1, pp. 25-34, 2002.
[19] J. A. Cramer, "Microelectronic systems for monitoring and enhancing patient compliance with medication regimens," Drugs, vol. 49, no. 3, pp. 321-327, 1995.

[20] D. P. Tashkin, C. Rand, M. Nides et al., "A nebulizer chronolog to monitor compliance with inhaler use," American Journal of Medicine, vol. 91, no. 4, pp. 33S-36S, 1991.

[21] B. Bender, F. Wamboldt, S. L. O’Connor et al., "Measurement of children's asthma medication adherence by self report, mother report, canister weight, and Doser CT, Annals of Allergy, Asthma and Immunology, vol. 85, no. 5, pp. 416-421, 2000.

[22] G. L. Braunstein, G. Trinquet, and A. E. Harper, "Compliance with nedocromil sodium and a nedocromil sodium/ salbutamol combination," European Respiratory Journal, vol. 9, no. 5, pp. 893-898, 1996.

[23] S. Hamid, J. Kumaradevan, and G. M. Cochrane, "Single centre open study to compare patient recording of PRN salbutamol use on a daily diary card with actual use as recorded by the MDI compliance monitor," Respiratory Medicine, vol. 92, no. 10, pp. 1188-1190, 1998.

[24] J. A. P. Coutts, N. A. Gibson, and J. Y. Paton, "Measuring compliance with inhaled medication in asthma," Archives of Disease in Childhood, vol. 67, no. 3, pp. 332-333, 1992.

[25] S. L. O'Connor, B. G. Bender, L. A. Gavin-Devitt et al., "Measuring adherence with the Doser CT in children with asthma," Journal of Asthma, vol. 41, no. 6, pp. 663-670, 2004.

[26] M. S. Simmons, M. A. Nides, C. S. Rand, R. A. Wise, and D. P. Tashkin, "Unpredictability of deception in compliance with physician-prescribed bronchodilator inhaler use in a clinical trial," Chest, vol. 118, no. 2, pp. 290-295, 2000.

[27] M. S. Simmons, M. A. Nides, C. S. Rand, R. A. Wise, and D. P. Tashkin, "Trends in compliance with bronchodilator inhaler use between follow-up visits in a clinical trial," Chest, vol. 109, no. 4, pp. 963-968, 1996.

[28] K. F. Chung and I. Naya, "Compliance with an oral asthma medication: a pilot study using an electronic monitoring device," Respiratory Medicine, vol. 94, no. 9, pp. 852-858, 2000.

[29] S. W. Burgess, P. D. Sly, A. Morawska, and S. G. Devadason, "Assessing adherence and factors associated with adherence in young children with asthma," Respirology, vol. 13, no. 4, pp. 559-563, 2008.

[30] C. L. M. Carnrike, L. M. McCracken, and J. E. Aikens, "Social desirability, perceived stress, and PACT ratings in lung transplant candidates: a preliminary investigation," Journal of Clinical Psychology in Medical Settings, vol. 3, no. 1, pp. 57-67, 1996.

[31] J. W. Finney, R. J. Hook, P. C. Friman, M. A. Rapoff, and E. R. Christophersen, "The overestimation of adherence to pediatric medical regimens," Children's Health Care, vol. 22, no. 4, pp. 297-304, 1993.

[32] C. M. Bosley, J. A. Fosbury, and G. M. Cochrane, "The psychological factors associated with poor compliance with treatment in asthma," European Respiratory Journal, vol. 8, no. 6, pp. 899-904, 1995.

[33] J. R. Gilbert, C. E. Evans, R. B. Haynes, and P. Tugwell, "Predicting compliance with a regimen of digoxin therapy in family practice," Canadian Medical Association journal, vol. 123, no. 2, pp. 119-122, 1980.

[34] A. I. Mushlin and F. A. Appel, "Diagnosing potential noncompliance. Physicians' ability in a behavioral dimension of medical care," Archives of Internal Medicine, vol. 137, no. 3, pp. 318-321, 1977. 
[35] N. A. Gibson, A. E. Ferguson, T. C. Aitchison, and J. Y. Paton, "Compliance with inhaled asthma medication in preschool children," Thorax, vol. 50, no. 12, pp. 1274-1279, 1995.

[36] B. Bender, H. Milgrom, and A. Apter, "Adherence intervention research: what have we learned and what do we do next?" Journal of Allergy and Clinical Immunology, vol. 112, no. 3, pp. 489-494, 2003.

[37] J. Reiser and J. O. Warner, "The value of participating in an asthma trial," The Lancet, vol. 1, no. 8422, pp. 206-207, 1985.

[38] E. Sumartojo, "When tubercolosis treatment fails: a social behavioral account of patient adherence," American Review of Respiratory Disease, vol. 147, no. 5, pp. 1311-1320, 1993.

[39] E. C. Perrin and J. M. Perrin, "Clinicians' assessments of children's understanding of illness," American Journal of Diseases of Children, vol. 137, no. 9, pp. 874-878, 1983.

[40] M. Bond, "Decision-making: risk school," Nature, vol. 461, no. 7268, pp. 1189-1192, 2009.

[41] B. Staples and T. Bravender, "Drug compliance in adolescents: assessing and managing modifiable risk factors," Pediatric Drugs, vol. 4, no. 8, pp. 503-513, 2002.

[42] E. L. McQuaid, S. J. Kopel, R. B. Klein, and G. K. Fritz, "Medication adherence in pediatric asthma: reasoning, responsibility, and behavior," Journal of Pediatric Psychology, vol. 28, no. 5, pp. 323-333, 2003.

[43] W. O. Cooper and G. B. Hickson, "Corticosteroid prescription filling for children covered by Medicaid following an emergency department visit or a hospitalization for asthma," Archives of Pediatrics and Adolescent Medicine, vol. 155, no. 10, pp. 1111-1115, 2001.

[44] G. Jónasson, K. H. Carlsen, A. Sødal, C. Jonasson, and P. Mowinckel, "Patient compliance in a clinical trial with inhaled budesonide in children with mild asthma," European Respiratory Journal, vol. 14, no. 1, pp. 150-154, 1999.

[45] C. S. Rand, "Adherence to asthma therapy in the preschool child," Allergy, vol. 57, no. 74, pp. 48-57, 2002.

[46] N. Walders, D. Drotar, and C. Kercsmar, "The allocation of family responsibility for asthma management tasks in AfricanAmerican adolescents," Journal of Asthma, vol. 37, no. 1, pp. 89-99, 2000.

[47] M. C. Mann, O. Eliasson, K. Patel, and R. L. ZuWallack, "An evaluation of severity-modulated compliance with q.i.d. dosing of inhaled beclomethasone," Chest, vol. 102, no. 5, pp. 1342-1346, 1992.

[48] J. V. Brown, E. Avery, C. Mobley, L. Boccuti, and T. Golbach, "Asthma management by preschool children and their families: a developmental framework," Journal of Asthma, vol. 33, no. 5, pp. 299-311, 1996.

[49] S. M. Penza-Clyve, C. Mansell, and E. L. McQuaid, "Why Don't children take their asthma medications? A qualitative analysis of children's perspectives on adherence," Journal of Asthma, vol. 41, no. 2, pp. 189-197, 2004.

[50] K. M. Buston and S. F. Wood, "Non-compliance amongst adolescents with asthma: listening to what they tell us about self-management," Family Practice, vol. 17, no. 2, pp. 134-138, 2000.

[51] H. Kyngäs, M. Hentinen, and J. H. Barlow, "Adolescents' perceptions of physicians, nurses, parents and friends: help or hindrance in compliance with diabetes self-care?" Journal of Advanced Nursing, vol. 27, no. 4, pp. 760-769, 1998.

[52] A. S. Kaugars, M. D. Klinnert, and B. G. Bender, "Family influences on pediatric asthma," Journal of Pediatric Psychology, vol. 29, no. 7, pp. 475-491, 2004.
[53] P. R. Wood, L. A. Smith, D. Romero, P. Bradshaw, P. H. Wise, and W. Chavkin, "Relationships between welfare status, health insurance status, and health and medical care among children with asthma," American Journal of Public Health, vol. 92, no. 9, pp. 1446-1452, 2002.

[54] S. J. Bartlett, J. A. Krishnan, K. A. Riekert, A. M. Butz, F. J. Malveaux, and C. S. Rand, "Maternal depressive symptoms and adherence to therapy in inner-city children with asthma," Pediatrics, vol. 113, no. 2, pp. 229-237, 2004.

[55] E. L. McQuaid, S. J. Kopel, and J. H. Nassau, "Behavioral adjustment in children with asthma: a meta-analysis," Journal of Developmental and Behavioral Pediatrics, vol. 22, no. 6, pp. 430-439, 2001.

[56] M. E. Christiaanse, J. V. Lavigne, and C. V. Lerner, "Psychosocial aspects of compliance in children and adolescents with asthma," Journal of Developmental and Behavioral Pediatrics, vol. 10, no. 2, pp. 75-80, 1989.

[57] S. L. Spector, R. Kinsman, H. Mawhinney et al., "Compliance of patients with asthma with an experimental aerosolized medication: implications for controlled clinical trials," Journal of Allergy and Clinical Immunology, vol. 77, no. 1, pp. 65-70, 1986.

[58] A. J. Apter, S. T. Reisine, G. Affleck, E. Barrows, and R. L. ZuWallack, "Adherence with twice-daily dosing of inhaled steroids: socioeconomic and health-belief differences," American Journal of Respiratory and Critical Care Medicine, vol. 157, no. 6, pp. 1810-1817, 1998.

[59] M. E. Mansour, B. P. Lanphear, and T. G. DeWitt, "Barriers to asthma care in urban children: parent perspectives," Pediatrics, vol. 106, no. 3, pp. 512-519, 2000.

[60] L. Irvine, I. K. Crombie, E. M. Alder, R. G. Neville, and R. A. Clark, "What predicts poor collection of medication among children with asthma? A case-control study," European Respiratory Journal, vol. 20, no. 6, pp. 1464-1469, 2002.

[61] J. Ho, B. G. Bender, L. A. Gavin, S. L. O’Connor, M. Z. Wamboldt, and F. S. Wamboldt, "Relations among asthma knowledge, treatment adherence, and outcome," Journal of Allergy and Clinical Immunology, vol. 111, no. 3, pp. 498-502, 2003.

[62] S. Hilton, H. R. Anderson, B. Sibbald, and P. Freeling, "Controlled evaluation of the effects of patient education on asthma morbidity in general practice," The Lancet, vol. 1, no. 8471, pp. 26-29, 1986.

[63] D. H. Rubin, L. J. Bauman, and J. L. Lauby, "The relationship between knowledge and reported behavior in childhood asthma," Journal of Developmental and Behavioral Pediatrics, vol. 10, no. 6, pp. 307-312, 1989.

[64] K. A. Riekert, A. M. Butz, P. A. Eggleston, K. Huss, M. Winkelstein, and C. S. Rand, "Caregiver-physician medication concordance and undertreatment of asthma among inner-city children," Pediatrics, vol. 111, no. 3, pp. e214-220, 2003.

[65] N. M. Clark, M. Gong, M. A. Schork et al., "Impact of education for physicians on patient outcomes," Pediatrics, vol. 101, no. 5, pp. 831-836, 1998.

[66] S. Iqbal, S. Ritson, I. Prince, J. Denyer, and M. L. Everard, "Drug delivery and adherence in young children," Pediatric Pulmonology, vol. 37, no. 4, pp. 311-317, 2004.

[67] S. P. Galant, J. Van Bavel, A. Finn et al., "Diskus and diskhaler: efficacy and safety of fluticasone propionate via two dry powder inhalers in subjects with mild-to-moderate persistent asthma," Annals of Allergy, Asthma and Immunology, vol. 82, no. 3, pp. 273-280, 1999. 
[68] S. W. Burgess, P. D. Sly, D. M. Cooper, and S. G. Devadason, "Novel spacer device does not improve adherence in childhood asthma," Pediatric Pulmonology, vol. 42, no. 8, pp. 736739, 2007.

[69] G. Chaney, B. Clements, L. Landau, M. Bulsara, and P. Watt, "A new asthma spacer device to improve compliance in children: a pilot study," Respirology, vol. 9, no. 4, pp. 499-506, 2004.

[70] M. Mann, O. Eliasson, K. Patel, and R. L. ZuWallack, "A comparison of the effects of bid and qid dosing on compliance with inhaled flunisolide," Chest, vol. 101, no. 2, pp. 496-499, 1992.

[71] H. Williams, E. R. V. Jones, and J. R. Sibert, "Twice daily versus four times daily treatment with beclomethasone dipropionate in the control of mild childhood asthma," Thorax, vol. 41, no. 8, pp. 602-605, 1986.

[72] C. Rand, A. Bilderback, K. Schiller, J. M. Edelman, C. M. Hustad, and R. S. Zeiger, "Adherence with montelukast or fluticasone in a long-term clinical trial: results from the mild asthma montelukast versus inhaled corticosteroid trial," Journal of Allergy and Clinical Immunology, vol. 119, no. 4, pp. 916-923, 2007.

[73] J. Sherman, P. Patel, A. Hutson, S. Chesrown, and L. Hendeles, "Adherence to oral montelukast and inhaled fluticasone in children with persistent asthma," Pharmacotherapy, vol. 21, no. 12, pp. 1464-1467, 2001.

[74] S. W. Stoloff, M. A. Stempel, J. Meyer, R. H. Stanford, and J. R. Carranza Rosenzweig, "Improved refill persistence with fluticasone propionate and salmeterol in a single inhaler compared with other controller therapies," Journal of Allergy and Clinical Immunology, vol. 113, no. 2, pp. 245-251, 2004.

[75] D. A. Stempel, S. W. Stoloff, J. R. C. Rosenzweig, R. H. Stanford, K. L. Ryskina, and A. P. Legorreta, "Adherence to asthma controller medication regimens," Respiratory Medicine, vol. 99, no. 10, pp. 1263-1267, 2005.

[76] M. P. Sovani, C. I. Whale, J. Oborne et al., "Poor adherence with inhaled corticosteroids for asthma: can using a single inhaler containing budesonide and formoterol help?" British Journal of General Practice, vol. 58, no. 546, pp. 37-43, 2008.

[77] M. Celano, R. J. Geller, K. M. Phillips, and R. Ziman, "Treatment adherence among low-income children with asthma," Journal of Pediatric Psychology, vol. 23, no. 6, pp. 345-349, 1998.

[78] T. Charles, D. Quinn, M. Weatherall, S. Aldington, R. Beasley, and S. Holt, "An audiovisual reminder function improves adherence with inhaled corticosteroid therapy in asthma," Journal of Allergy and Clinical Immunology, vol. 119, no. 4, pp. 811-816, 2007.

[79] M. A. Nides, D. P. Tashkin, M. S. Simmons, R. A. Wise, V. C. $\mathrm{Li}$, and C. S. Rand, "Improving inhaler adherence in a clinical trial through the use of the nebulizer chronolog," Chest, vol. 104, no. 2, pp. 501-507, 1993.

[80] F. Onyirimba, A. Apter, S. Reisine et al., "Direct clinician-topatient feedback discussion of inhaled steroid use: its effect on adherence," Annals of Allergy, Asthma and Immunology, vol. 90, no. 4, pp. 411-415, 2003.

[81] S. J. Bartlett, P. Lukk, A. Butz, F. Lampros-Klein, and C. S. Rand, "Enhancing medication adherence among inner-city children with asthma: results from pilot studies," Journal of Asthma, vol. 39, no. 1, pp. 47-54, 2002.

[82] S. W. Burgess, P. D. Sly, and S. G. Devadason, "Providing feedback on adherence increases use of preventive medication by asthmatic children," Journal of Asthma, vol. 47, no. 2, pp. 198-201, 2010. 


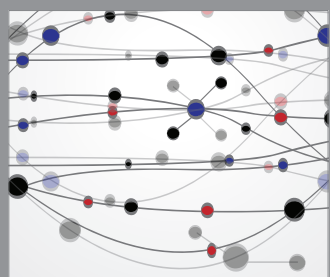

The Scientific World Journal
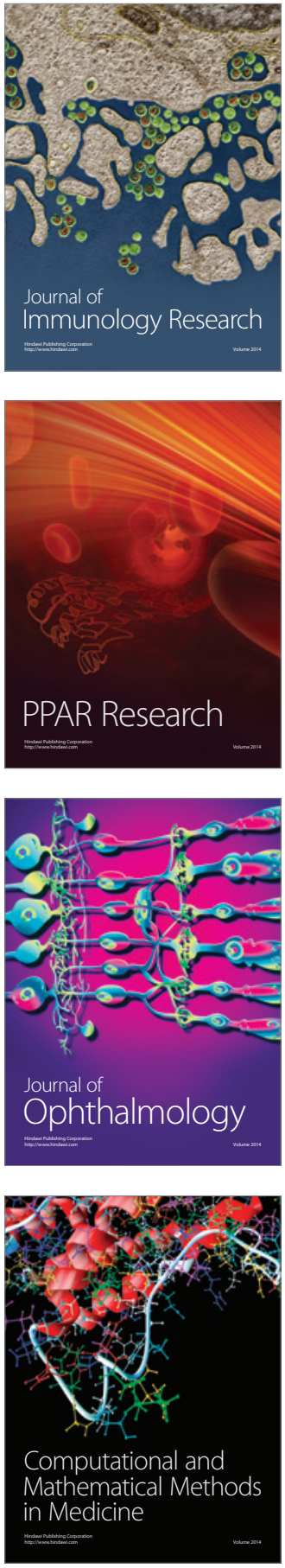

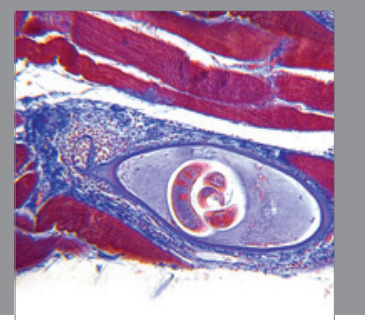

Gastroenterology

Research and Practice
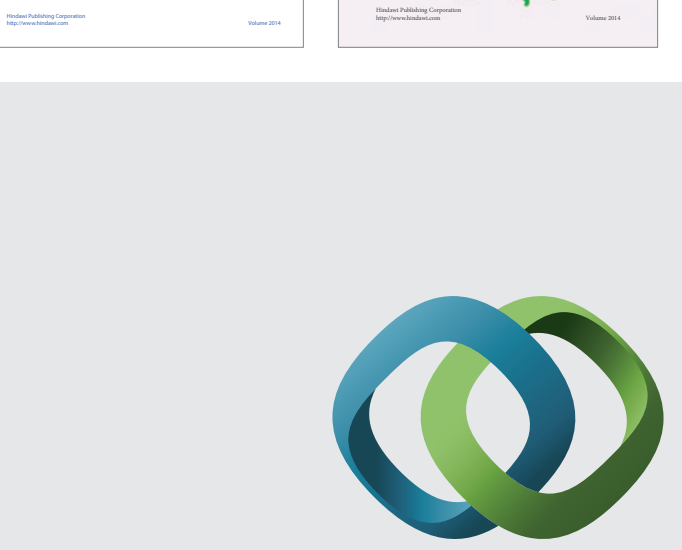

\section{Hindawi}

Submit your manuscripts at

http://www.hindawi.com
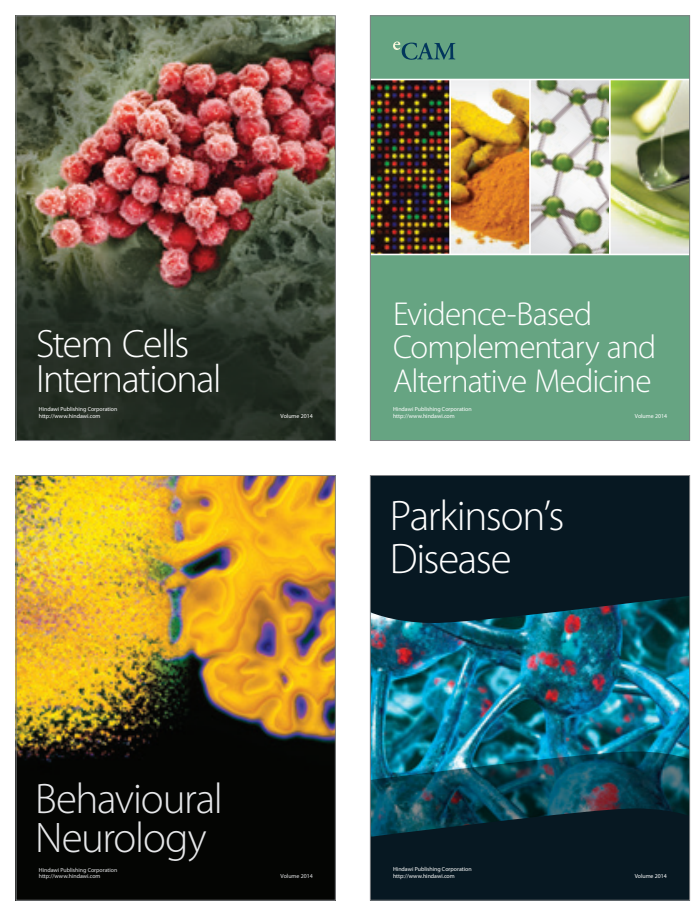

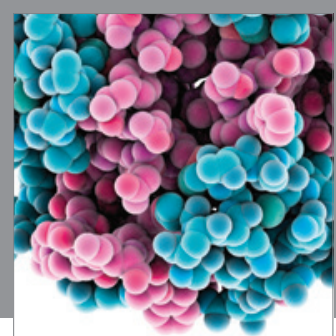

Journal of
Diabetes Research

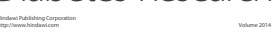

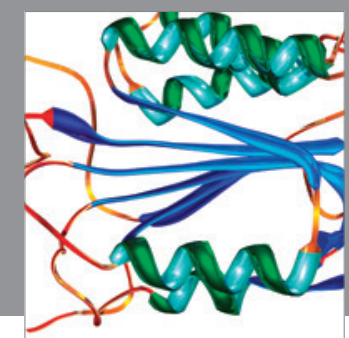

Disease Markers
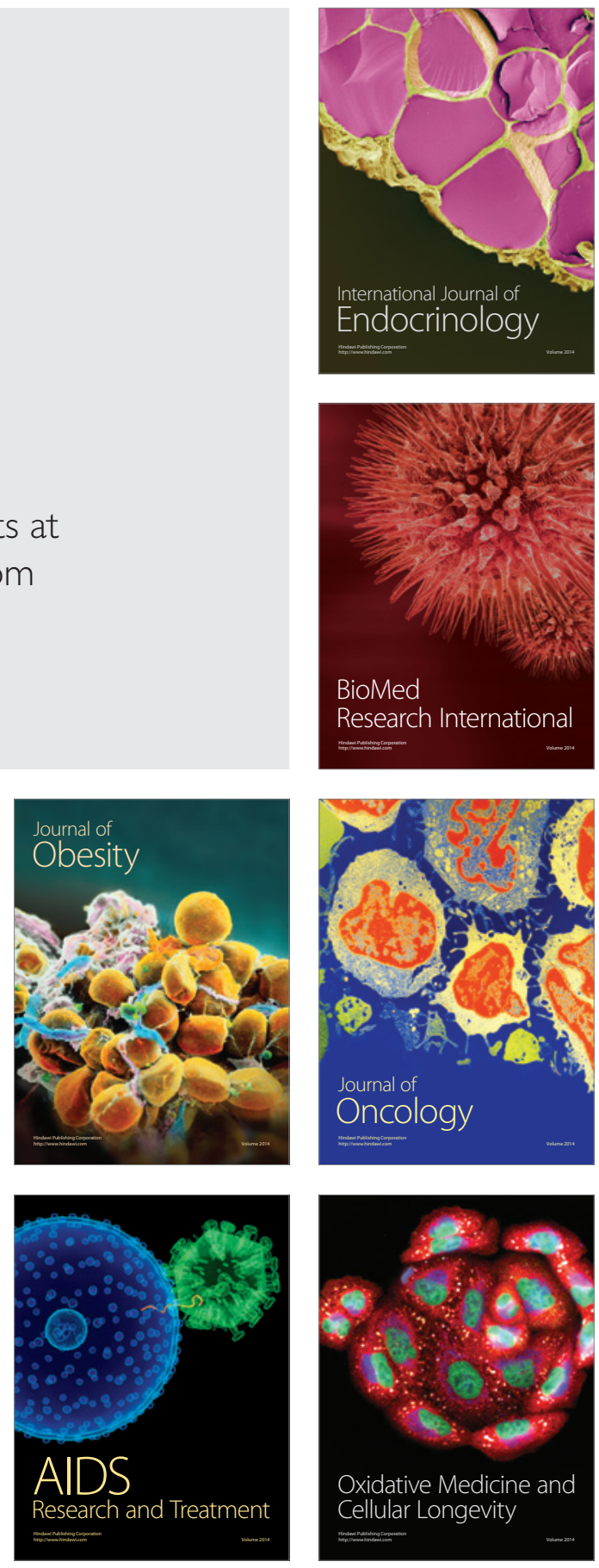